\title{
Anaphylaxis in Children: Experience of an Egyptian Center
}

\author{
Ghada Shousha $^{1}$, MOHAMED EZZAT $^{1}$, Hebat-Allah Gabal ${ }^{1}$, and Mousa Mousa ${ }^{2}$ \\ ${ }^{1}$ Ain Shams University Faculty of Medicine \\ ${ }^{2}$ Sirte University Faculty of Medicine
}

December 1, 2020

\begin{abstract}
Introduction: Anaphylaxis is a potentially life threatening allergic reaction that is rapid in onset and multisystemic in nature. Distribution of anaphylaxis tends to fluctuate based on age, gender, race, geographical residence and socioeconomic status of the involved subjects. Diagnosis of anaphylaxis in children is generally underestimated particularly in developing countries, and when diagnosed, proper management is occasionally lacking. Aim of the study: to evaluate the frequency and pattern of anaphylaxis and mistakes in diagnosis and treatment among a group of Egyptian children and adolescents. Subjects and methods: This observational study was conducted over 6 months duration, from 1st of September 2019 to the end of February 2020, in Children's hospital, Ain Shams University, Cairo, Egypt, on children presenting to emergency department (ED). Anaphylaxis frequency, presentation, triggers, diagnosis, management and long term follow up were assessed. Results: frequency rate of anaphylaxis among children and adolescents was $0.43 \%(80 / 18816)$ over a year. Sixty percent of patients were infants. Biphasic reactions were reported in $11.3 \%$ of patients. The commonest trigger was food $(22.5 \%)$ followed by medications $(8.8 \%)$. Epinephrine was given to all patients, in proper dosage and method in most occasions (78.7\%). Long term management and follow up appointment were deficient. In conclusion: Anaphylaxis in children was relatively high, with food being the most common trigger. Diagnosis and early management of anaphylaxis was satisfactory, however, long term management and follow up were defective.
\end{abstract}

Title Page

Title of the Manuscript:

Anaphylaxis in Children: Experience of an Egyptian Center

Running head title: Anaphylaxis in Children

Key words: Anaphylaxis, Biphasic reactions, Epinephrine, Triggers,

Number of Tables: 3

Number of Figures: 4

Number of References: 30

No conflicts of interest are to be declared. The research was not financially funded.

\section{Abstract}

Introduction: Anaphylaxis is a potentially life threatening allergic reaction that is rapid in onset and multisystemic in nature. Distribution of anaphylaxis tends to fluctuate based on age, gender, race, geographical residence and socioeconomic status of the involved subjects. Diagnosis of anaphylaxis in children 
is generally underestimated particularly in developing countries, and when diagnosed, proper management is occasionally lacking.Aim of the study: to evaluate the frequency and pattern of anaphylaxis and mistakes in diagnosis and treatment among a group of Egyptian children and adolescents.Subjects and methods: This observational study was conducted over 6 months duration, from $1^{\text {st }}$ of September 2019 to the end of February 2020, in Children's hospital, Ain Shams University, Cairo, Egypt, on children presenting to emergency department (ED). Anaphylaxis frequency, presentation, triggers, diagnosis, management and long term follow up were assessed.Results: frequency rate of anaphylaxis among children and adolescents was $0.43 \%$ (80/18816) over a year. Sixty percent of patients were infants. Biphasic reactions were reported in $11.3 \%$ of patients. The commonest trigger was food $(22.5 \%)$ followed by medications $(8.8 \%)$. Epinephrine was given to all patients, in proper dosage and method in most occasions (78.7\%). Long term management and follow up appointment were deficient.In conclusion: Anaphylaxis in children was relatively high, with food being the most common trigger. Diagnosis and early management of anaphylaxis was satisfactory, however, long term management and follow up were defective.Key statement: Anaphylaxis is generally underdiagnosed and maltreated particularly in children owing to the lack of data and awareness among physicians as well as general population. In this work we spotted the light on the frequency of anaphylaxis in children in one of the highly rated University Hospitals in Egypt, and the most suspected triggers, clinical presentation and steps of management.IntroductionAnaphylaxis is a severe, life-threatening, multisystemic hypersensitivity reaction. Although it occurs in patients of all ages, most research and guidelines are focused on adults. Elicitors and clinical presentation of anaphylaxis in children and adolescents were shown to be different from those in adults, calling for specific research for pediatric populations ${ }^{1}$. Guidelines for both adults and children stress on rapid diagnosis as being a key to optimal management. Although cutaneous symptoms predominate in adults, the primary presenting symptoms in children are respiratory in nature (e.g., wheezing, shortness of breath). In addition, cardiovascular symptoms tend to be less common in children than in adults. Moreover, food related causes, which tend to cause respiratory manifestations, are more common in children whereas medication and venom triggers, which tend to cause cardiovascular reactions, are more common in adults ${ }^{2}$. The incidence of anaphylaxis is underestimated in various studies owing to the problems of recognizing it and the variability of criteria of diagnosis in different studies and countries ${ }^{3}$. The American Academy of Allergy, Asthma and Immunology (AAAAI) Epidemiology of Anaphylaxis Working Group concluded that the overall frequency of anaphylaxis ranges between 30 and 950 cases per 100,000 persons per year and lifetime prevalence 50 - 2000 episodes per 100,000 persons $(0.05-2 \%)^{4}$. In children, the most common food allergens are milk products, peanuts, tree nuts, eggs, shellfish, and fruits and vegetables ${ }^{5}$. Biphasic allergic reactions are reported in 1 to $23 \%$ of anaphylaxis attacks particularly in patients with severe initial episodes or those who were not given epinephrine in the early phase of anaphylaxis ${ }^{6}$. Factors that increase the risk of severe or fatal anaphylactic episodes are similar worldwide. They include age-related factors, concomitant diseases such as asthma and other chronic respiratory diseases, cardiovascular diseases, mastocytosis or clonal mast cell disorders, and severe atopic disease. Some concurrent medications like beta adrenergic blockers and ACE inhibitors might also potentiate the risk ${ }^{7}$. The overall prognosis of anaphylaxis is good. Early injection of epinephrine in anaphylaxis (defined as injection before arrival at ED) can significantly reduce the likelihood of hospital admission. Delayed injection of epinephrine has been reported in a large case series of anaphylaxis-related fatalities ${ }^{8}$. Mistakes in the diagnosis of anaphylaxis may occur because of the limited time during which the diagnosis must be made, the stressful environment of the emergency room, the incomplete clinical features in early anaphylaxis and the lack of useful laboratory markers.Several studies have shown that anaphylaxis is often miscoded or misclassified. The frequency of miscoding appears to be similar in adults and children ${ }^{9}$. Updated World Allergy Organization (WAO) anaphylaxis guidelines highlighted the importance of epinephrine being the only effective treatment and that there is no role for glucocorticoids or antihistamines in severe allergic reactions ${ }^{10}$. International research agenda for anaphylaxis was concerned with the unmet needs in anaphylaxis in high, mid and low income countries ${ }^{11}$. To our knowledge, there are few registries concerning anaphylaxis in Egypt, therefore we aimed to evaluate the frequency and pattern of anaphylaxis and mistakes in diagnosis and treatment among a group of Egyptian children and adolescents presented to Children's hospital, Ain Shams University, Cairo, Egypt.Patients and Methods:This observational cross-sectional study was conducted on all the children and adolescents 
who presented to ED of Children's hospital, Ain Shams University, Cairo, Egypt, in the period from 1st of September 2019 to the end of February 2020. All patients were examined properly by the investigator for possibility of anaphylaxis diagnosis based on the updated criteria settled by WAO at 2011. Diagnosis of anaphylaxis was declared when the patient fulfilled one of the following scenarios ${ }^{12}: \mathrm{I}_{-}$Sudden onset (minutes to several hours) of muco-cutaneous manifestations involving skin, mucosal tissue or both (generalized hives, urticaria, pruritus and flushing, swollen lips, tongue or uvula) and at least one of the followings: ${ }^{*}$ Respiratory compromise (bronchospasm, wheeze, stridor, hypoxemia, reduced peak expiratory flow). * Reduced blood pressure (BP) or associated symptoms and signs of end-organ dysfunction (collapse, syncope, incontinence). II-Two or more of the following that occur rapidly after exposure to a likely allergen for that patient (minutes to several hours): * Muco-cutaneous manifestations as described above. * Respiratory compromise as described above. ${ }^{*}$ Reduced BP or associated signs and symptoms of end-organ dysfunction. * Persistent gastrointestinal signs and symptoms (crampy abdominal pain, nausea, vomiting or diarrhea). III- Reduced BP after exposure to a known allergen for that patient (minutes to several hours): * Reduced BP in adults is defined as a systolic BP (SBP) of less than $90 \mathrm{mmHg}$ or $30 \%$ decrease in that patient's baseline. ${ }^{*}$ In infants and children, reduced BP is defined as low systolic BP (age specific) or greater than $30 \%$ decrease in SBP.Exclusion criteria:

Patients with neurological illness affecting their conscious level

Patients with congenital heart diseases because of the possible confusing symptoms with anaphylaxis

All children presented to ED (except those in the exclusion criteria) during the duration of the study were examined for anaphylaxis. Those who fulfilled the criteria of diagnosis were recruited in the study and observed properly until they were sent home.Methods:All the included patients were subjected to the following:

Detailed history taking: including socio-demographic data, personal or family history of atopy, previous severe allergic reactions/anaphylaxis, their description and management.

Description of the current anaphylactic reaction through history and examination as regards:

- Presenting symptoms and signs

- Possible culprit trigger

- The initial diagnosis at ED

- The initial management of anaphylaxis at ED

- Second line medications given for the patients

- Long term management of anaphylaxis including patient and family education, providing a written plan, prescribing epinephrine as needed and arranging a follow up appointment at the Allergy clinic in Children's hospital, Ain Shams University.

The researcher introduced himself to parents of all participants in this study and written consent was taken; however, he did not interfere with the management provided for those patients. Ethical committee approval was gained prior to the start of this work. The collected data was revised, coded, tabulated and introduced to a PC using Statistical Program for Social Science version 22 (SPSS Inc., Chicago, IL, USA, 2001). Data were presented and suitable analysis was done according to the type of data obtained for each parameter.Results:We defined 80 patients with anaphylaxis out of 18816 representing all the ED visitors at the period of the study with frequency rate $0.43 \%$. The studied children were $47(58.8 \%)$ males and 33 (41.3\%) females with mean age $2.94 \pm 3.44$ years. Age of affected patients varied widely with the majority being infants. Age distribution is shown in figure1. Unexpectedly, most of patients were not known to be atopic. The atopic patients were suffering mainly of food allergy and allergic rhinitis, followed by bronchial asthma and eczema; with family history of atopy in more than half of those atopic patients. Only one patient had a previous attack of anaphylaxis (table1). The culprit trigger was unidentified in more than half of patients. However, food was the most offending identified trigger followed by medications (figure2). Drug-induced anaphylaxis represented $8.8 \%$ of the studied cases, divided between antibiotics $(57.14 \%)$ and NSAID (42.86\%). Age groups that suffered from drug-induced anaphylaxis were infants $(71.4 \%)$ followed by 
preschoolers (28.6\%). Non-steroid anti-inflammatory drugs (NSAIDs) allergy was limited to infants while antibiotics induced anaphylaxis was equally distributed between infants and Pre-school age groups as shown in figure3. Signs and symptoms of anaphylaxis at time of presentation were variable among children. Urticarial hives and facial edema were the most common presenting symptom followed by respiratory symptoms (dyspnea, cough, wheezes) and gastrointestinal symptoms (nausea/vomiting, abdominal cramps). Two patients out of 80 had hypotension at time of presentation (table2). Biphasic reaction was reported in $11.3 \%$ of patients within 6-8 hours after the first attack, distributed in different age groups as follows: infants (6.3\%), preschoolers (11.8\%), schoolers (33.3\%) and none of the adolescents. All patients presented with anaphylaxis were correctly diagnosed in ED of our University hospital, thus all of them were provided intramuscular epinephrine given in the anterolateral aspect of the thigh, calculated in the correct dose; however, $21.3 \%$ of patients were given diluted (1:10000) epinephrine instead of concentrated shots (figure4). More than one shot of epinephrine was needed in $22.5 \%$ of patients. One patient had protracted anaphylaxis that necessitated ICU admission and intravenous infusion of epinephrine. As regards the outcome of those patients, the majority were stabilized after the initial management and sent home, 2 patients were admitted to hospital (drug-induced anaphylaxis) and the patient who experienced protracted anaphylaxis, has passed away (table3). Second line treatment was given to all patients in the form of glucocorticoids and anti-histamines type1. Unfortunately, long term management was defective. None of the patients were given a written plan for possible future anaphylactic reactions, prescribed epinephrine for emergencies or scheduled for an Allergy Clinic visit for follow up and further management.

\section{Discussion:}

In this study, we have identified 80 patients with anaphylactic reactions out of 18816 over 6 months with frequency rate $0.43 \%$. This frequency rate is relatively higher than what was reported in different studies and this might be attributed to the rarity of centers having specialized Allergy units in Egypt, in addition to the University hospital being a tertiary referral one. Anagnostou and colleagues, as an example, reported an incidence rate of 50-112 per 100000 (0.05-0.112\% per year); and estimated lifetime prevalence of anaphylaxis between $0.5 \%-2 \%^{13}$. A meta-analysis, done on 59 studies conducted on children, estimated that incidence rate of anaphylaxis was ranging from 1 to 761 per 100000-year (0.001-0.76\%); however, studies from developed countries were underrepresented ${ }^{6}$. The European anaphylaxis registry has identified 1970 patients with anaphylaxis aged younger than 18 years in the duration between July 2007 and March $2015^{5}$. In an Irish study conducted over 4 years on children aged 14 years old and lower, the number of children with at least one anaphylactic reaction was $63^{14}$. A prospective study from Denmark conducted on children and adults has identified 180 anaphylactic patients over a year representing $0.3-0.4 \%$ of all contacts in ED with children's incidence rate of 26.8 per 100000 year $(0.027 \%)^{15}$. A Korean single center study has recorded 107 children with anaphylaxis over 3 years ${ }^{16}$. Males were more represented in this study than females with the majority of patients being infants. Anaphylaxis was reported in higher rates in males in more than one study $^{5,6,14,16}$. It was noticeable in many studies that anaphylaxis is more prevalent in children compared with adults, however, the reactions used to be milder ${ }^{5,6}$. Castells and colleagues stated that severe reactions are 9 -fold for adults compared with children ${ }^{17}$. We noticed in this study that frequency rate of anaphylaxis decreased with age' being higher-most in infants and lowermost in adolescents, however, the study duration and population were relatively limited being a single center. Wide based studies like the European anaphylaxis registry, has collected data from 90 study center representing 10 countries and they reported, on the contrary, lowermost cases in the infants and highest incidence in the preschool children followed by school aged then adolescents ${ }^{5}$. Atopy as a risk factor of anaphylaxis and a determinant factor of severity of episodes was reported in less than one third of our studied children and more than half of their families with food allergy and allergic rhinitis being the most frequently documented. Atopy was repeatedly visualized as a risk factor that might worsen the clinical outcome of anaphylaxis ${ }^{19}$. Abunada and colleagues showed that personal history of atopy for asthma, atopic dermatitis and allergic rhinitis were noted among patients with anaphylaxis; and $56.9 \%$ of patients had positive family history which matches our findings ${ }^{20}$. Although a few studies showed no such significant association between atopy and anaphylaxis, several studies stated that anaphylaxis was common among atopic patients ${ }^{5,16}$. Triggers of anaphylaxis varied widely. On the top of 
list was food allergens followed by drug and insect bites, albeit unidentified triggers were reported in more than half of the studied patients. It was agreed worldwide, based on findings reported by many studies, that food allergens were the most common triggers of anaphylaxis in children in contrast to adults whereas druginduced anaphylaxis comes first ${ }^{5,6,14,16}$. In our study, $8.8 \%$ of anaphylactic children were triggered by drugs, mainly antibiotics and NSAIDs with the latter being more obvious in the infantile age group. We had lost one patient out of anaphylaxis which was antibiotic induced. Retrigo and colleagues documented drug triggered anaphylaxis in $3.1 \%$ of preschoolers, $4.1 \%$ of school aged and $12.1 \%$ of adolescents with analgesics being on the top of list ${ }^{21}$. It is to be noted that majority of anaphylaxis fatalities reported in other studies were caused by medications; with antibiotics and analgesics carrying the highest risk ${ }^{20,21}$. Obviously, presenting signs and symptoms of anaphylaxis were widely variable. Cutaneous manifestations were the most common followed by gastrointestinal and respiratory ones. Only two patients out of 80 were hypotensive. This wide variation in the clinical presentation of anaphylaxis was documented in many single and multi-center studies with the age being the most determinant factor ${ }^{5,22}$. In the current research, pruritus and gastrointestinal symptoms were more dominant in the infants and young children. On the other hand, respiratory symptoms were more obvious in older children and adolescents. This agrees with the reported findings of many other studies $^{5,14,16,23}$. Biphasic reaction was recorded in $11.3 \%$ of our studied patients being more common in scholar age group followed by preschoolers. The severity of those reactions was generally mild and occurred within 6 to 8 hours after the first attack being more evident in those patients who presented late to ED and hence were delayed in receiving epinephrine. Compared to other studies, we recorded a relatively high rate of biphasic reactions. Jeon and colleagues reported biphasic reaction in $4.7 \%$ of their studied patients ${ }^{24}$. A study in Thailand documented $6.3 \%$ of the anaphylaxis patients experienced biphasic reaction and that the time interval from onset to administration of epinephrine was a predicator ${ }^{25}$. A multi-institution research on pediatric anaphylaxis in Turkey reported biphasic reaction in $3.1 \%$ of anaphylactic patients ${ }^{26}$. Milder or similar second attacks of anaphylaxis in children were documented in many studies ${ }^{23}$. As known, diagnosis of anaphylaxis is a challenge owing to the wide differential diagnoses and similarities in manifestations. Indeed, errors in diagnosing and managing anaphylaxis by health providers and junior medical staff are a documented problem worldwide ${ }^{25-27}$. Fortunately, in this study, all patients with anaphylaxis were properly diagnosed and treated with epinephrine, although less than quarter of them was given epinephrine in improper way, being diluted instead of concentrated shots. Epinephrine is a life-saving medication and proper use during an anaphylactic reaction is of paramount importance. In a study done by Castilano and colleagues in one of the university hospitals in USA, they found that only $20 \%$ of those diagnosed as anaphylaxis in ED were given intramuscular epinephrine, thus many patients needed hospital admission. One of the reported barriers to the proper use of epinephrine is lack of proper training with regard to the correct technique ${ }^{27}$. Oropeza and colleagues multicenter research recorded that only $25 \%$ of patients with anaphylaxis were given epinephrine ${ }^{15}$. European anaphylaxis registry has documented treatment with epinephrine at ED in only $28 \%$ of anaphylactic patients ${ }^{5}$. Another study conducted in Australia on anaphylaxis in children reported missed diagnosis of anaphylaxis in about $50 \%$ of patients fulfilling the criteria and that $85.7 \%$ of those who were confirmed had received epinephrine injection ${ }^{28}$. Lee and colleagues found that $71 \%$ of the patients with anaphylaxis were given IM epinephrine ${ }^{16}$. Second line medications in the form of glucocorticoids and type1 antihistamines were supplied to all the studied patients. Although they have no role in the management of anaphylaxis as recommended by $\mathrm{WAO}^{10}$ and National Institute of Allergy, Immunology, and Infectious Diseases (NIAID) guidelines ${ }^{29}$, glucocorticoids and histamine1 blockers were commonly prescribed to control the muco-cutaneous manifestations and to reduce hospitalization of anaphylactic patients ${ }^{30}$. Unfortunately, long term management was not provided to any of our studied patients including proper family education or scheduled appointment for follow up in the Specialized Allergy Clinic in our University hospital. The latter was partly attributed to COVID19 pandemic which lead to strict regulations of follow up. The lack of providing parents with adequate clarification and education about anaphylaxis will definitely lead to increased morbidities and mortalities. This problem was reported in many studies particularly not providing a written anaphylaxis action plan ${ }^{16,27}$. In conclusion, anaphylaxis is a growing problem and it is relatively high in children particularly in infants with food being the most common trigger. Diagnosis and early management of anaphylaxis was satisfactory, however, long term management, follow up and patient education still needs 
many efforts. A wide scale registry of anaphylaxis from different governments of Egypt is recommended. Educational sessions should be designed to healthcare providers for better orientation about the proper management of anaphylaxis and the paramount importance of patient and family education.References:

1. Kim SY, Kim MH, Cho YJ. Different clinical features of anaphylaxis according to cause and risk factors for severe reactions. Allergol Intern. 2018; 67(1):96-102.

2. Simons FE, Sampson HA. Anaphylaxis: unique aspects of clinical diagnosis and management in infants (birth to age 2 years). J Allergy Clin Immunol. 2015; 135(5): 1125-31.

3. Grattan CE, Borzova E. Urticaria, Angioedema, and Anaphylaxis. In Clinic Immunol ELSEVEIR. 2019; 11: 585-600.

4. Joyce EY, Lin RY. The epidemiology of anaphylaxis. Clin Rev Allerg Immun. 2018; 54(3): 366-74.

5. Sicherer SH, Sampson HA. Food allergy: a review and update on epidemiology, pathogenesis, diagnosis, prevention, and management. J Allergy Clin Immun. 2018; 141(1): 41-58.

6. Grabenhenrich LB, Dolle S, Moneret-Vautrin A, Kohli A, Lange L, Spindler T, et al. Anaphylaxis in children and adolescents: the European Anaphylaxis Registry. J Allergy Clin Immun. 2016; 137(4): 1128-37.

7. Wang Y, Allen KJ, Suaini NHA, McWilliam V, Peters RL, Koplin JJ. The global incidence and prevalence of anaphylaxis in children in the general population: a systematic review. Allergy. 2019; 74(6):1063-80.

8. Sanchez-Borges M, Aberer W, Brockow K, Celik GE, Cernadas J, Greenberger PA, et al. Trautmann A. Controversies in drug allergy: radiographic contrast media. J Allergy Clin Immun: In Practice. 2019; 7(1): 61-5.

9. Brown JC, Simons E, Rudders SA. Epinephrine in the Management of Anaphylaxis. J Allergy Clin Immunol. 2020; 8(4):1186-95.

10. De Feo G, Parente R, Triggiani M. Pitfalls in anaphylaxis. Curr Opin Allergy Clin Immun. 2018; 18: 382-6.

11. Shaker MS, Wallace DV, Golden DB, Oppenheimer J, Bernstein JA, Campbell RC, et al. Anaphylaxisa 2020 Practice Parameter Update, Systematic Review and GRADE Analysis. J Allergy Clin Immunol. 2020; 28: 142-53.

12. Simons FE, Ardusso LR, Bilo MB, El_Gamal YM, Ledford DK, Ring J et al. World allergy organization guidelines for the assessment and management of anaphylaxis. J Allergy Clin Immunol. 2011; 7:587-93.

13. Hayden ML. Review of Controversial Areas in Anaphylaxis for the Nurse Practitioner. J Nurse Pract. 2018; 14(7): 531-7.

14. Anagnostou K. Anaphylaxis in Children: Epidemiology, Risk Factors and Management. Current Pediatric Reviews. 2018; 14(3).

15. Barzegar S, Rosita A, Pourpak Z, Bemanian MH, Shokouhi R, Mansouri M et al. Common causes of anaphylaxis in children: the first report of anaphylaxis registry in Iran. World Allergy Organ J. 2020; 3(1): 9-13.

16. Oropeza AR, Lassen A, Halken S, Bindslev_Jensen C, Mortz CG. Anaphylaxis in an emergency care setting: a one year prospective study in children and adults. Scand J Trauma Resusc Emerg Med. 2017; 25(11). https://doi.org/10.1186/s13049-017-0402-0

17. Lee WS, An J, Jung YH, Jee HM, Chae KY, Park YA et al. Characteristics and treatment of anaphylaxis in children visiting a pediatric emergency department in Korea. BioMed Res Int. 2020. https://doi.org/10.1155/2020/2014104

18. Castells M. Diagnosis and management of anaphylaxis in precision medicine. J Allergy Clin Immunol. 2017; 140(2): 321-33.

19. Jares EJ, Baena-Cagnani CE, Sanchez-Borges M, Ensina LF, Arias-Cruz A, Gomez M, et al. Druginduced anaphylaxis in Latin American countries. J Allergy Clin Immunol Pract. 2015; 3(5): 780-8.

20. Abunada T, Al-Nesf MA, Thalib L, Kurdi R, Khalil S, ElKassem W, et al. Anaphylaxis triggers in a large tertiary care hospital in Qatar: a retrospective study. World Allergy Organ J. 2018; 11(1): 20-9.

21. Retrigo FS, Marques ML, Gomes ER. Drug induced anaphylaxis: an update on epidemiology and risk factors. Int Arch Allergy Immunol. 2020; 181:481-7. 
22. Rudders SA, Banerji A, Clark S, Camargo CA Jr. Age-related differences in the clinical presentation of food-induced anaphylaxis. J Pediatr. 2011; 158(2):326-8.

23. Scranton SE, Gonzalez EG, Waibel KH. Incidence and characteristics of biphasic reactions after allergen immunotherapy. J. Allergy Clin. Immunol. 2009; 123:493- 8.

24. Jeon YH, Lee S, Ahn K, Lee SY, Kim KW, Kim HH, et al. Infantile anaphylaxis in Korea: a multicenter retrospective case study. J Korean Med Sci. 2019; 34(13): 222-34.

25. Lertnawapan R, Maek NW. Anaphylaxis and biphasic phase in Thailand: 4-year observation. Allergol Int. 2011; 60(3):283-9.

26. Orhan F, Canitez Y, Bakirtas A, Yilmaz O, Boz AB, Can D, et al. Anaphylaxis in Turkish children: a multi-centre, retrospective, case study. Clin Exp Allergy. 2011; 41(12):1767-76.

27. Prince BT, Mikhail I, Stukus DR. Underuse of epinephrine for the treatment of anaphylaxis: missed opportunities. J Asthma Allergy. 2018; 11: 143-51.

28. Thomson H, Seith R, Craig S. Downstream consequences of diagnostic error in pediatric anaphylaxis. BMC Pediatr. 2018; 18:40.

29. Won L, Jaewoo A, Young-Ho J, Hye J, Kyu-Young C, Young P, et al. Characteristics and Treatment of Anaphylaxis in Children Visiting a Pediatric Emergency Department in Korea. BioMed Res Intern. 2020. 1-7. 10.1155/2020/2014104.

30. Shiratori T, Sato A, Fukuzawa M, Kondo N, Tanno S. Severe dextran-induced anaphylactic shock during induction of hypertension-hypervolemia-hemodilution therapy following subarachnoid hemorrhage. Case Reports in Critical Care. 2015; 1:5.

\section{Hosted file}

Tables.pdf available at https://authorea.com/users/380223/articles/496303-anaphylaxis-inchildren-experience-of-an-egyptian-center

\section{Hosted file}

Figures.pdf available at https://authorea.com/users/380223/articles/496303-anaphylaxis-inchildren-experience-of-an-egyptian-center 